\author{
Historical Studies in Education / Revue d'histoire de l'éducation \\ SECTION THÉMATIQUE I / SPECIAL FEATURE I \\ L'éducation francophone en Ontario / French Language Education in Ontario
}

\title{
La conscience historique de jeunes franco-ontariens d'ottawa : histoire et sentiment $d^{\prime}$ appartenance ${ }^{1}$
}

\author{
Stéphane Lévesque, Jean-Philippe Croteau et Raphaël Gani
}

\begin{abstract}
RÉSUMÉ
Les écoles de langue française de l'Ontario inscrivent au cœur de leur projet éducatif la mission de transmettre aux élèves francophones une culture commune et un patrimoine historique et de favoriser leur appartenance à l'identité franco-ontarienne. Cet article vise à vérifier si l'Ontario français constitue un point d'ancrage dans la conscience historique des jeunes francophones et s'ils ont recours au passé pour se forger une identité de citoyen. Nous avons mené une enquête dans deux écoles secondaires d'Ottawa et dans une classe de didactique de l'histoire à l'Université d'Ottawa, où les élèves et les futurs enseignants devaient indiquer leur degré d'attachement identitaire et rédiger un récit sur l'histoire l'Ontario. Il en résulte qu'une appartenance forte à l'identité franco-ontarienne amène les jeunes à puiser dans le passé de l'Ontario français pour consolider et renforcer cette identité dans leur conscience de citoyen. À l'opposé, une identité canadienne et ontarienne forte se traduit pour les élèves par un récit moins engagé politiquement et plus descriptif centré sur l'histoire de son pays ou de sa province.
\end{abstract}

\section{ABSTRACT}

A central pillar of French-language schooling in Ontario is the mission to transmit a shared culture and history to Francophone students, and encourage their attachment to Franco-Ontarian identity. This article aims to verify whether French Ontario is an important component of the historical consciousness of young Francophones, and if they turn to the past to forge their identities as citizens. Students in two Ottawa secondary schools and future teachers in a history education class at the University of Ottawa were asked to indicate their degree of attachment to Franco-Ontarian identity and to create an account of Ontario history. We found that a strong sense of belonging as Franco-Ontarians leads young people to look to the past to help them construct and reinforce their identity as citizens. On the other hand, strong Canadian and Ontarian identities are expressed by students in more descriptive-and less politically engaged - accounts of the history of their homeland or province.

L'histoire est un puissant catalyseur pour stimuler le sentiment d'appartenance identitaire au sein d'un groupe culturel et le cas des Franco-Ontariens est révélateur à ce sujet. La fête du drapeau franco-ontarien et les Monuments de la francophonie qui 
jalonnent le territoire ontarien ont pour fonction de renforcer le sentiment d'appartenance et d'éveiller la fierté vis-à-vis d'un parcours historique qui se veut singulier. Le système scolaire de langue française exerce une fonction similaire avec le cours d'histoire de l'Ontario français, dispensé en $12^{\mathrm{e}}$ année. Celui-ci conduit les élèves à étudier "les origines, l'évolution et le maintien culturel de l'Ontario français, du XVII siècle jusqu'à nos jours. " ${ }^{2}$ Ce cours est en continuité avec les travaux d'historiens qui, depuis les années 1970, rendent compte des efforts des francophones de l'Ontario à "faire communauté " en reconstituant une genèse qui fait remonter la présence française ou même "franco-ontarienne " au régime français, soit le début du XVII ${ }^{\mathrm{e}}$ siècle. Cette genèse se caractérise notamment par la prévalence des luttes politiques et scolaires pour le fait français qui sont érigés en mythes fondateurs. ${ }^{3}$

Parmi ces luttes scolaires et politiques, notons les crises du Règlement XVII et de l'hôpital Montfort qui ont joué un rôle marquant dans l'identité franco-ontarienne et ce, encore aujourd'hui. La première crise renvoie à l'adoption par le gouvernement conservateur Whitney, en 1912, d'un règlement pour proscrire l'enseignement du français dans les écoles bilingues de l'Ontario. Une politique abandonnée progressivement à partir de la fin des années 1920 après une campagne menée par l'Association canadienne-française d'éducation (ACFÉO) qui prit différentes formes (désobéissance civile, luttes devant les tribunaux, négociations avec le gouvernement, etc.). SOS Montfort a été un mouvement de résistance mené en 1997 par la mairesse de Vanier, Gisèle Lalonde, pour empêcher la fermeture par le gouvernement conservateur Harris du seul hôpital bilingue à l'ouest du Québec. La Cour d'appel de l'Ontario a donné raison au mouvement SOS Montfort reconnaissant aux francophones en Ontario le droit à leurs institutions. ${ }^{4}$ Les récits historiques de l'Ontario français n’hésitent pas à faire le parallèle entre les deux événements qui représentent la volonté collective du peuple franco-ontarien de perpétuer la culture et la langue française. Ils se manifestent dans les programmes scolaires mais aussi dans les productions artistiques comme la reconstitution historique à grand déploiement "L'Écho d'un peuple ", dont le succès ne s'est jamais démenti en accueillant plus de 100000 spectateurs entre 2004 et $2008 .^{5}$

Peu de chercheurs ont tenté de vérifier le postulat voulant que l'adhésion au récit historique de l'Ontario français soit liée au sentiment d'appartenance franco-ontarien. Ainsi, il nous apparaît primordial d'évaluer ce lien dans un contexte où la communauté francophone de l'Ontario connaît une reconfiguration de ses paramètres identitaires et culturels avec notamment l'arrivée d'immigrants francophones surtout depuis les années 1990.

Comme l'a bien montré Anne Gilbert, l'Ontario français est divisée en différents espaces qui constituent des milieux de vie francophone. ${ }^{6}$ Bien qu'Ottawa ne soit pas nécessairement représentatif de l'ensemble des communautés francophones de l'Ontario, il reste que cette ville et les environs exercent une influence importante en Ontario français en raison de ses caractéristiques géographiques, démographiques, culturelles et socioéconomiques. D'emblée, Ottawa se caractérise par un bassin de population de langue française important. Près d'un francophone de l'Ontario sur quatre vit à Ottawa. Cette concentration de la population francophone dans 
la région d'Ottawa lui a permis d'atteindre la complétude institutionnelle et de conserver un des taux de rétention linguistique les plus élevés dans la province. ${ }^{7}$ De plus, la proximité du Québec insuffle un dynamisme sans précédent à la vie institutionnelle, culturelle et communautaire francophone d'Ottawa qui consolide la structuration d'un espace francophone grâce à un apport migratoire quotidien ou permanent. La présence de l'administration publique fédérale - mais aussi provinciale et municipale dans une moindre mesure - dans la région d'Ottawa a permis aux francophones de faire fructifier leur capital linguistique en occupant des emplois bilingues. De fait, les francophones d'Ottawa ont un taux de chômage plus bas et des revenus moyens et médians plus élevés que le reste de la population régionale et provinciale, de même que la population francophone des autres régions de l'Ontario. Enfin, la population francophone d'Ottawa connaît un essor important grâce à l'immigration qui en fait avec Toronto le siège du pluralisme culturel de la francophonie ontarienne. ${ }^{8}$ Ainsi, pour certains, la ville d'Ottawa représente la " capitale de l'Ontario français. " Dans le cas qui nous préoccupe, elle constitue un terrain d'analyse privilégié pour notre recherche exploratoire sur le lien qui unit le sentiment d'appartenance envers la communauté franco-ontarienne et l'adhésion au récit historique de l'Ontario français.

\section{L'école et l'histoire franco-ontarienne : son rôle dans la (re) production du sentiment d'appartenance}

Dès le départ, l'Ontario français s'est inscrit au cœur du projet éducatif des écoles de langue française de l'Ontario, dont l'une des missions est de transmettre aux élèves francophones une culture et un patrimoine historique commun afin de favoriser une identité franco-ontarienne. Cette vision de l'éducation s'est réaffirmée avec la réforme des programmes scolaires ontariens en 1999 à la suite de la Commission royale d'enquête sur l'éducation ontarienne, intitulée " Pour l'amour d'apprendre ». ${ }^{10}$ Ainsi, il nous a semblé opportun de mener notre étude exploratoire au sein d'écoles francophones de la région d'Ottawa, afin d'observer la manière dont les élèves s'approprient ce qui leur est enseigné au sujet de l'Ontario français.

En raison des caractéristiques sociodémographiques du milieu de vie francophone d'Ottawa, il nous apparait qu'une étude au sein des écoles secondaires de cette ville permet de rendre compte du processus de construction identitaire que connaissent les jeunes franco-ontariens. Nous sommes toutefois conscients du fait que la capitale fédérale n'est pas représentative des communautés francophones de l'Ontario qui, à l'image de la population ontarienne en générale, est diverse, vibrante et répartie dans plusieurs régions de la province. En effet, la complétude institutionnelle d'Ottawa, son bassin de population francophone important, la proximité du Québec et la place occupée par la fonction publique fédérale dans l'économie de la région constituent des facteurs qui favorisent l'épanouissement culturel et identitaire de la minorité qui dispose d'un plus grand capital symbolique, social et culturel que d'autres régions francophones.

Bien que le caractère représentatif de notre échantillon peut être mis en doute surtout par rapport au reste de la province, nous concevons que le principal intérêt 
d'une enquête dans le milieu scolaire franco-ontarien d'Ottawa réside dans le fait qu'il représente à long terme ce à quoi pourrait ressembler la nouvelle francophonie ontarienne, alors que l'immigration devient un phénomène incontournable et ce, dans plusieurs communautés de la province. Entre Toronto, porte d'entrée de l'immigration francophone et d'autres régions plus homogènes culturellement comme le Nord-Est ou l'Est ontarien, Ottawa semble occuper une position intermédiaire en jouant un rôle grandissant comme pôle d'attraction des nouveaux arrivants. Dans ces circonstances, il est d'autant plus pertinent d'étudier la région d'Ottawa, car elle nous permet de valider si les programmes d'études remplissent les objectifs du ministère de l'Éducation et parviennent à inculquer et transmettre un sentiment d'appartenance identitaire à l'Ontario français aux élèves au secondaire et aux futurs enseignants.

À l'heure actuelle, l'Ontario français est un concept largement utilisé en éducation à des fins d'instruction et de socialisation. ${ }^{11}$ Il fait partie des orientations des nouveaux programmes-cadres du ministère de l'Éducation de l'Ontario et s'inscrit directement dans les divers objectifs d'apprentissage en histoire et en études sociales. Ainsi, en études sociales ( $1^{\mathrm{re}}-6^{\mathrm{e}}$ année), on indique aux enseignants que " les activités et le matériel d'apprentissage à l'appui du curriculum doivent refléter la diversité de la communauté francophone de l'Ontario et de la société ontarienne dans son ensemble ${ }^{12}$ De manière plus explicite, le cours d'histoire du Canada (10e année) amène l'élève à étudier des " activités de coopération et conflits qui se sont produits au Canada et en Ontario français " ${ }^{13}$ On invite notamment l'élève à " décrire la contribution de personnes, d'institutions et d'organismes au patrimoine culturel des francophones de l'Ontario (p. ex., Napoléon Belcourt, Jeanne Lajoie, Samuel Genest, lutte contre le Règlement 17); famille, paroisse, école maintien de la cohésion sociale; Association canadienne-française d'éducation d'Ontario (ACFÉO), défense des droits juridiques des francophones, Fédération des femmes canadiennes-françaises (FFCF) à sa création en 1918, œuvre de bienfaisance venant en aide aux Canadiennes et Canadiens français ». ${ }^{14}$ De manière plus marquée, le programme-cadre de $12^{\mathrm{e}}$ année inclut un cours optionnel intitulé "L'Ontario français " ayant pour objectif d'amener l'élève à étudier " les origines, l'évolution et le maintien culturel de l'Ontario français, du XVII siècle jusqu'à nos jours, et en retracer l'histoire par rapport à celle du Canada, de l'Amérique du Nord et du monde. ${ }^{15}$. Toutefois, l'offre de cours varie considérablement d'une école à l'autre en fonction de la demande. Ainsi, sa portée demeure limitée puisqu'il ne rejoint qu'un nombre restreint d'élèves francophones dans la province.

\section{Postulats de recherche}

Bien que le rapport à l'histoire chez les Franco-Ontariens soit un thème de plus en plus privilégié par les chercheurs, la revue de la littérature est plutôt mince à propos de la place de l'Ontario français dans la " conscience historique » des jeunes francophones. ${ }^{16}$ On peut concevoir la conscience historique comme la capacité d'appréhender et de comprendre — de manière formelle et informelle — des informations et 
des expériences diverses touchant le passé « dès lors portées à un niveau secondaire d'assimilation et d'appropriation $"{ }^{17}$ La conscience historique permet à tout individu de s'orienter dans le temps et ainsi de développer son identité-personnelle et collective - à titre de citoyen et qui mobilise ses connaissances historiques pour l'action notamment sous forme de récits qui lient le passé au présent et à l'avenir possible. En ce sens, la conscience historique est davantage que la mémoire historique qui découle de ce qu'un individu ou une collectivité retient et transmet - consciemment ou inconsciemment — du passé. Alors que la mémoire est constituée de savoirs entassés soumis à la dialectique du rappel et de l'oubli, la conscience historique est une sorte d'intellection, de conceptualisation du passé à des fins d'orientation dans le temps. ${ }^{18}$

Comme nous l'avons vu, les programmes scolaires des écoles de langue française visent à inculquer aux jeunes un sentiment d'appartenance à la communauté francoontarienne. Plus particulièrement, les programmes en histoire sont censés jouer un rôle déterminant dans ce processus identitaire. Toutefois, aucune donnée de recherche ne nous permet actuellement de corroborer l'affirmation voulant qu'à la suite de leur passage dans une école de langue française, les jeunes francophones intègrent l'Ontario français dans leur conscience historique. De plus, nous en savons peu sur le lien qui unit le sentiment d'appartenance à la communauté franco-ontarienne et l'adhésion au récit historique de l'Ontario français. Le sentiment d'appartenance à la communauté des Franco-Ontariens influence-t-il l'adhésion à une lecture particulière de l'histoire de l'Ontario français ? Observe-t-on une vision du passé divergente entre ceux qui s'identifient fortement ou faiblement à la communauté franco-ontarienne?

Cette recherche de nature exploratoire a pour objet d'étude de vérifier la prégnance et l'enracinement de l'Ontario français dans la conscience historique des jeunes franco-ontariens. Il s'inscrit dans une perspective plus globale visant à sonder la conscience historique des jeunes francophones de la province. Il nous permet de rendre compte des résultats initiaux du projet franco-ontarien pour ainsi mieux baliser les prochaines étapes de notre étude à l'échelle provinciale. Dans cette recherche, nous nous sommes intéressés tout particulièrement au lien entre l'appartenance à la communauté franco-ontarienne et le récit historique des jeunes francophones issus d'écoles de la région d'Ottawa. Rappelons que le sentiment d'appartenance se définit par une appréciation subjective du lien qui unit un individu à son groupe d'appartenance.

De plus, nous souhaitons évaluer le rôle de l'école dans la (re) production identitaire et dans la formation de la conscience historique. Est-ce que la conscience historique des élèves reflète les programmes d'études enseignés à l'école ou n'est-ce pas plutôt le milieu social qui agit comme un curriculum réel d'expériences multiples et qui forge la conscience historique de l'élève en dépit des visées de l'école? ${ }^{19}$ Est-ce que le milieu social et culturel hétérogène façonne chez les jeunes un imaginaire historique en rupture avec celui présenté par l'école? Est-ce que plusieurs récits historiques cohabitent ou s'affrontent au sein des mêmes groupes de jeunes?

Nous avons donc postulé dans ce projet pilote que le sentiment d'appartenance à la communauté franco-ontarienne peut avoir un effet mobilisateur les amenant à privilégier un récit historique engagé politiquement. Ce récit engagé retrace, entre 
autres, les étapes d'un parcours historique caractérisé par les luttes politiques et les résistances visant la survie du fait français en Ontario.

\section{Méthodologie}

Pour évaluer si l'Ontario français constitue un point d'ancrage dans la conscience historique des Franco-Ontariens, nous nous sommes inspirés de la méthode employée par Jocelyn Létourneau et Marc Robichaud dans leurs travaux respectifs sur la conscience historique des jeunes québécois et des jeunes acadiens. ${ }^{20}$ Leurs travaux ont privilégié une approche novatrice qui consiste à demander aux élèves de rédiger un récit historique de leur communauté (une histoire). S'en suit une analyse des réponses qui a pour but de cartographier les différentes visions du passé exprimées la plupart du temps sous forme de narration. ${ }^{21}$ Ces études ont relevé que les adolescents québécois et acadiens ont des rapports complexes avec le passé. Ces derniers disposent d'une conscience historique ancrée dans leur société d'appartenance qui s'intègre à leur récit d'histoire, celui-ci étant caractérisé par sa cohérence et sa structure - ce qui n'exclut pas «les clichés, stéréotypes et mythistoires".

Pour cette première phase de notre recherche, nous avons constitué un échantillon de 58 élèves de $11^{\mathrm{e}}$ et $12^{\mathrm{e}}$ année dans deux écoles secondaires de la région d'Ottawa. Les classes de niveau supérieur comportent un intérêt tout particulier puisque les élèves de ce niveau ont complété l'ensemble de leur cursus scolaire obligatoire. Dans le curriculum ontarien, c'est en $7^{\mathrm{e}}, 8^{\mathrm{e}}$, et $10^{\mathrm{e}}$ année que les élèves suivent obligatoirement des cours d'histoire du Canada, présentés chronologiquement de la colonisation jusqu'à nos jours. C'est également au niveau supérieur que les adolescents intériorisent les connaissances historiques enseignées en classe et les intègrent dans leur identité grâce au processus de socialisation qui se fait parallèlement. Enfin, c'est en $11^{\mathrm{e}}$ et $12^{\mathrm{e}}$ année que certains élèves choisissent de poursuivre leurs études historiques notamment au sein du cours CHF4O l'Ontario français. Malheureusement, ce cours à option n'est pas offert dans toutes les écoles de l'Ontario compte tenu du peu d'inscriptions. Aucun élève n'avait suivi ce cours dans les écoles de notre recherche.

De manière plus spécifique, notre échantillon d'élèves du secondaire est composé de 36 élèves nés en Ontario, dont 34 à Ottawa, de 10 jeunes nés au Québec, d'un jeune provenant de Vancouver, d'un autre du "Canada " et le reste $(n=9)$ provenant de l'extérieur du pays, dont trois de la République démocratique du Congo et deux de Djibouti. Un jeune n'a pas indiqué son lieu de naissance. En ce qui a trait de l'origine ethnique, 16 élèves ont deux parents d'origine canadienne. Ensuite, 41 jeunes ont au moins un parent originaire de l'extérieur du Canada; un élève a indiqué "blanc " comme origine ethnique de ses parents. Pour la langue maternelle, 36 élèves ont le français comme langue maternelle, sept jeunes ont l'anglais et un seul élève a indiqué l'anglais et le français. Ensuite, 14 élèves ont une langue maternelle " autre ", dont le créole et l'arabe. Pour ce qui est de la langue parlée à la maison, 16 jeunes parlent le français à la maison, neuf parlent l'anglais et 18 jeunes parlent l'anglais et le français. Enfin, 15 jeunes emploient au moins une langue qui n'est pas le français ou l'anglais. Rappelons que cet échantillon n'est pas représentatif de l'ensemble de 
la population franco-ontarienne, mais il constitue néanmoins un groupement beaucoup plus typique de la nouvelle jeunesse francophone et des nouvelles tendances sociodémographiques qui façonnent à l'heure actuelle la collectivité franco-ontarienne au sein du système scolaire de l'Ontario et de la région d'Ottawa.

À l'hiver 2014, l'équipe de recherche a invité les élèves volontaires à répondre à la directive suivante: "Raconte-moi l'histoire de l'Ontario comme tu la connais ". ${ }^{22} \mathrm{Au}$ cours d'une période de 60 minutes, les élèves devaient rédiger leur réponse à l'ordinateur sans limites d'espace ou de structure de présentation et sous le couvert de la confidentialité.

Pour mieux analyser les sources d'inspiration et d'influence des jeunes, nous sommes allés au delà du récit afin de poser certaines questions biographiques et démographiques (âge, sexe, lieu de naissance, origine ethnique, langue maternelle et parlée à la maison et avec les amis, etc.). Enfin, les élèves devaient définir en identifiant des cercles concentriques leur degré d'appartenance aux identités canadienne, ontarienne et franco-ontarienne. ${ }^{23}$

En plus de notre échantillon d'élèves du secondaire, le même questionnaire a été soumis auprès de 18 futurs enseignants de l'Université d'Ottawa qui ont reçu une formation en histoire et en didactique de l'histoire. ${ }^{24}$ Cet échantillon a été divisé en deux groupes (split sample). Le premier groupe devait répondre à la question, "Raconte-moi l'histoire de l'Ontario français comme tu la connais", tandis que le second groupe devait répondre à la question : "Raconte-moi l'histoire de l'Ontario comme tu la connais". Ce procédé visait à vérifier si on pouvait observer des recoupements ou des juxtapositions entre les récits des deux questions (Ontario français vs Ontario) ou, au contraire, si ces deux récits produisaient des narrations complètement distinctes. Bien que distincts, nous sommes d'avis que nos deux échantillons sont pertinents pour cette étude exploratoire, car ils servent d'abord à étudier les parallèles et les contrastes entre les visions historiques de jeunes provenant du système scolaire francophone et de futurs enseignants qui ont acquis un bagage historique et disciplinaire plus étendu. Ces échantillons permettent ensuite de mieux comprendre l'importance de la formation académique, ainsi que le rôle des expériences de vie formatrices sur la conscience historique des francophones vivant à Ottawa au moment de l'étude. Bref, l'utilisation de ces deux échantillons donne plus d'épaisseur, de richesse et de contexte à la notre étude exploratoire sur la conscience historique.

L'analyse des données, tant pour les élèves du secondaire que les futurs enseignants d'histoire, s'articulent autour de deux axes : la manière dont ils évaluent leur appartenance à la communauté franco-ontarienne et les mentions factuelles et organisationnelles utilisées pour raconter l'histoire de l'Ontario. ${ }^{25}$ Les mentions factuelles et organisationnelles du récit narratif des jeunes franco-ontariens, calculées et tabulées sous forme de tableaux et de pourcentage, renvoient aux dates, événements, personnages, lieux, institutions ou organisations cités par les jeunes. Ces éléments en disent long sur le sens interprétatif que l'élève prête au parcours historique de sa collectivité. En croisant les données émanant de l'évaluation du sentiment d'appartenance avec celles concernant les mentions factuelles et organisationnelles des récits d'histoire, nous avons tenté d'établir le lien entre l'appartenance 
identitaire des élèves et leur conscience historique. ${ }^{26}$ Pour y arriver, nous avons fait appel au logiciel QDA Miner, un logiciel d'analyse reconnu et très puissant, qui permet d'obtenir des listes de fréquence liée aux codes et aux mots-clés, d'identifier les relations entre les codes et d'évaluer la relation possible entre les codes et certaines variables telles que le sentiment d'appartenance. Il est important de souligner que les extraits des récits provenant des élèves et des futurs enseignants de notre étude sont présentés dans leur version originale sans égard aux erreurs d'orthographe, de grammaire et de syntaxe.

\section{Résultats}

Le premier élément qui ressort des résultats est la différence marquée entre les récits des élèves du secondaire et ceux des futurs enseignants. Ces derniers sont beaucoup plus riches en contenu avec une moyenne de 462 mots par récit comparativement à 107 mots chez ceux du secondaire. Les futurs enseignants ont donc produit dans l'ensemble des récits quatre fois plus longs, souvent balisés par une trame chronologique détaillée et une vision plus engagée de l'histoire. Comme on pouvait s'y attendre, les futurs enseignants disposent sans l'ombre d'un doute d'un bagage historique beaucoup plus étendu que les élèves de $11^{\mathrm{e}}$ et $12^{\mathrm{e}}$ année avec, à leur crédit, plusieurs cours d'histoire de niveau universitaire. La différence est d'autant plus importante que ces futurs enseignants ont consciemment choisi de s'inscrire dans un programme de formation à l'enseignement de l'histoire. Pour toutes ces raisons, les réponses des futurs enseignants constituent un corpus tout à fait particulier qu'il nous incombe d'analyser de manière distincte pour ensuite voir comment ces récits nous sont utiles pour comprendre ceux des élèves du secondaire.

\section{Le récit des futurs enseignants : des passeurs de l'identité franco-ontarienne?}

Étudier le récit historique des futurs enseignants d'histoire constitue un point de départ important, car ceux-ci représentent l'avenir de l'éducation historique de langue française en Ontario. L'une de leurs tâches sera de développer chez leurs élèves une appartenance identitaire à la communauté franco-ontarienne, ainsi qu'à la culture francophone. Dans ce contexte, il est pertinent de se poser la question du récit historique que privilégient ces futurs enseignants pour comprendre la ou les conception(s) de l'aventure franco-ontarienne qu'ils valoriseront et qu'ils pourront transposer dans leur enseignement de l'histoire. Rappelons que l'expérience avec les futurs enseignants a constitué un premier essai et que ceux-ci furent divisés en deux groupes : l'un devait raconter l'histoire de l'Ontario français et l'autre groupe a disserté sur l'histoire de l'Ontario.

D’emblée, il ressort très clairement de cette enquête que : 1) L'Ontario français est davantage marqueur de sens que l'est l'Ontario. 2) Concernant la question générale sur l'histoire de l'Ontario, plusieurs étudiants ont adopté une perspective identitaire franco-ontarienne, c'est-à-dire qu'ils ont privilégié la description de l'Ontario français plutôt que de la province en général. Ainsi, l'histoire de l'Ontario peut 
servir de passerelle aux participants pour raconter l'histoire de l'Ontario français, alors que l'inverse est rare. Enfin, 3) neuf participants (futurs enseignants) originaires du Québec ont éprouvé de la difficulté à produire un récit sur l'Ontario et l'Ontario français bien qu'un certain nombre d'entre eux se destinent à enseigner au sein d'écoles franco-ontariennes.

Quelle vision les futurs enseignants offrent-ils de l'histoire de l'Ontario et de l'Ontario français? Le tableau 1 présente les mentions factuelles et organisationnelles qui structurent leurs récits. Les pourcentages représentent le nombre de participants qui ont fait référence aux mentions factuelles par rapport au nombre total d'universitaires interrogés, soit 18. Comme on peut le constater, les termes qui reviennent le plus souvent chez les futurs enseignants sont : l'Ontario (100\%), le Québec (94\%), le Canada (89 \%), les Français (89 \%), le Haut-Canada (83 \%), le Bas-Canada (72\%), la Conquête (72\%), la langue (67\%), les Anglais (61\%) et les Franco-Ontariens (61\%). On retrouve ensuite les mentions à Ottawa (56\%), aux Loyalistes $(50 \%)$ au pouvoir (50\%), au droit (50\%), aux écoles francophones $(50 \%)$ et au Règlement XVII (44\%). Moins fréquents, mais tout de même significatifs à la série événementielle des récits, se trouvent les mots-clés Autochtones (39\%), Confédération (39\%), guerre d'indépendance américaine (39\%), Étienne Brûlé (39\%), Champlain (22\%) et Montfort (17\%). Tous ces référents historiques sont davantage que de simples mots clés choisis au hasard par les futurs enseignants. Ces mots s'inscrivent au sein d'une trame narrative et donc, par le fait même, acquièrent une signification toute particulière. Ainsi, grâce à ce codage, on peut remarquer une certaine logique prénarrative qui met en relief des conflits historiques (Conquête, Règlement XVII, Rapport Durham, Montfort) entre groupements identitaires (Français, Anglais, Franco-Ontariens, Loyalistes, Autochtones) au sein de territoires politiques déterminés (Haut-Canada, Bas-Canada, Canada, États-Unis, Québec).

Tableau 1

Mentions factuelles dans les récits selon le pourcentage de cas

\begin{tabular}{|c|c|}
\hline Mots employés à l'université & $\begin{array}{c}\text { Pourcentage de cas } \\
\text { (n=18 futurs enseignants) }\end{array}$ \\
\hline ONTARIO & $100 \%$ \\
\hline QUÉBEC & $94 \%$ \\
\hline CANADA & $89 \%$ \\
\hline FRANÇAIS & $89 \%$ \\
\hline HISTOIRE & $83 \%$ \\
\hline HAUT-CANADA & $83 \%$ \\
\hline FRANCOPHONE & $78 \%$ \\
\hline BAS-CANADA & $72 \%$ \\
\hline CONOUÊTE & $72 \%$ \\
\hline TERRITOIRE & $67 \%$ \\
\hline
\end{tabular}




\begin{tabular}{|c|c|}
\hline Mots employés à l'université & $\begin{array}{c}\text { Pourcentage de cas } \\
\text { ( } n=18 \text { futurs enseignants) }\end{array}$ \\
\hline LANGUE & $67 \%$ \\
\hline ANGLAIS & $61 \%$ \\
\hline FRANCO (ONTARIEN) & $61 \%$ \\
\hline GUERRE & $56 \%$ \\
\hline OTTAWA & $56 \%$ \\
\hline ANGLOPHONE & $56 \%$ \\
\hline POUVOIR & $56 \%$ \\
\hline PAYS & $50 \%$ \\
\hline NOUVELLE-FRANCE & $50 \%$ \\
\hline LOYALISTES & $50 \%$ \\
\hline DROIT & $50 \%$ \\
\hline ÉCOLE & $50 \%$ \\
\hline RAPPORT DURHAM & $50 \%$ \\
\hline RÈGLEMENT XVII & $44 \%$ \\
\hline AMÉRICAIN & $44 \%$ \\
\hline ÉTIENNE BRÛLÉ & $39 \%$ \\
\hline CULTURE & $39 \%$ \\
\hline COLONIE & $39 \%$ \\
\hline AUTOCHTONES & $39 \%$ \\
\hline 1867/CONFÉDÉRATION & $39 \%$ \\
\hline GUERRE D'INDÉPENDANCE (USA) & $39 \%$ \\
\hline CAPITALE & $28 \%$ \\
\hline COMMUNAUTÉ & $28 \%$ \\
\hline CHAMPLAIN & $22 \%$ \\
\hline TERRE & $17 \%$ \\
\hline MONTFORT & $17 \%$ \\
\hline
\end{tabular}

Chez nos participants universitaires, l'attachement à une communauté d'appartenance influence-t-il les événements historiques choisis pour raconter l'histoire de l'Ontario ou de l'Ontario français? A ce jour, peu de données permettent de répondre à cette question. Pour y arriver, nous avons donc analysé les mots clés (du tableau 1) en relation avec la question demandant aux participants d'identifier leur appartenance à la communauté franco-ontarienne. Deux éléments ressortent de cette analyse croisée.

D'abord, les participants s'identifiant fortement à la communauté franco-ontarienne $(\mathrm{N}=8)$ ont produit des récits légèrement plus longs que ceux qui s'identifient 
moyennement ou faiblement à cette communauté. Ainsi, les futurs enseignants qui s'identifient fortement au Franco-Ontarien utilisent en moyenne 484 mots. À l'inverse, ceux qui s'identifient moyennement ou peu à cette communauté ont produit des récits contenant 422 mots en moyenne. C'est donc dire que les participants avec un fort attachement à la communauté franco-ontarienne ont tendance à produire des récits plus riches en contenu. En fait, la deuxième découverte de notre analyse traite justement de ce contenu.

Comme l'indique le tableau 2, certains mots tirés des récits sont fortement associés au niveau d'identification. Ainsi, les participants s'identifiant fortement à la communauté franco-ontarienne ont davantage utilisé les termes "communauté ", "Français ", " lutte " " école " et " Règlement ". Ils favorisent généralement un récit dualiste qui présente un peuple en marche pour la conquête de ses droits, ponctué de moments de luttes et de résistance. À l'inverse, les mots clés " colonie ", " population » et "Canada » sont davantage utilisés par les participants s’identifiant moyennement ou peu à cette communauté. Ces derniers privilégient un récit historique plus général et descriptif (dates, lieux, événements) qui confond l'Ontario et le Canada et évacue généralement de leur trame narrative les conflits linguistiques :

À travers le temps, l'Ontario a su se tailler une place importante au sein du Canada. Il s'agit d'une province riche en ressources naturelles. Cette province détient un grand pouvoir économique et est une des provinces qui contribue le plus à l'économie du pays. (participant 019)

C'est donc dire, encore ici, que les contenus factuels retenus par les futurs enseignants dans leurs récits s'inscrivent dans une certaine logique narrative, notamment chez ceux qui s'identifient fortement comme Franco-Ontariens, qui met en lumière une communauté d'appartenance particulière en lutte pour sa survie et qui s'appuie, entre autres, sur le système scolaire comme instrument d'éducation, de socialisation et de reproduction culturelle :

En 1912, l'Ontario français subi le plus grand choque de son histoire; la crise du Règlement XVII. Établi par le gouvernement ontarien de James P. Whitney, le Règlement XVII interdit l'enseignement et l'usage du français dans les écoles de l'Ontario. On assistera donc à un élan de mobilisation de la part de la communauté franco-ontarienne. Il y aura des épisodes héroïques, tels que la bataille des épingles à chapeaux. Mais, nous retrouverons de nouveaux héros de l'histoire franco-ontarienne; de personnes telles que Jeanne Lajoie, Béatrice Desloges, Samuel Genest, etc. Par la suite, l'Ontario français devra se défendre à plusieurs reprises pour la survie de sa langue et sa culture. Il s'agit de conflits tels que pour la création de nos écoles secondaire (on pense ici à Sturgeon Falls et Penetangueshene), la création de nos collèges, et également à SOS Montfort. (participant 003) 
Tableau 2

Nombre de mentions thématiques

dans les récits en nombre selon l'identité des participants

\begin{tabular}{|c|c|c|c|}
\hline Mots clés & $\begin{array}{l}\text { Identité franco- } \\
\text { ontarienne forte }(n=8)^{1}\end{array}$ & $\begin{array}{l}\text { Identité franco- } \\
\text { ontarienne moyenne à } \\
\text { faible }(n=9)\end{array}$ & $\begin{array}{l}\text { Identification } \\
\text { prédominante }\end{array}$ \\
\hline COMMUNAUTÉ & 18 & 1 & Forte \\
\hline COLONIE & 2 & 17 & $\begin{array}{l}\text { Moyenne } \\
\text { à faible }\end{array}$ \\
\hline FRANÇAIS & 52 & 30 & Forte \\
\hline AUTOCHTONE & 9 & 1 & Forte \\
\hline LUTTE & 12 & 4 & Forte \\
\hline ÉCOLE & 16 & 7 & Forte \\
\hline FRANCO & 17 & 8 & Forte \\
\hline ONTARIENNE & 8 & 2 & Forte \\
\hline DROIT & 12 & 5 & Forte \\
\hline ÉDUCATION & 9 & 3 & Forte \\
\hline RÈGLEMENT & 10 & 4 & Forte \\
\hline POPULATION & 4 & 12 & $\begin{array}{l}\text { Moyenne } \\
\text { à faible }\end{array}$ \\
\hline ANGLAIS & 16 & 9 & Forte \\
\hline ONTARIO & 52 & 42 & Forte \\
\hline CATHOLIQUE & 9 & 4 & Forte \\
\hline OTTAWA & 10 & 5 & Forte \\
\hline LANGUE & 12 & 7 & Forte \\
\hline CANADA & 32 & 47 & $\begin{array}{l}\text { Moyenne } \\
\text { à faible }\end{array}$ \\
\hline FRANCOPHONE & 26 & 21 & Forte \\
\hline
\end{tabular}

1. Le nombre total de réponses n'arrive pas à 18 car un des participants universitaires n'a pas complété la section du questionnaire traitant de l'identité.

Observation intéressante, plusieurs participants qui s'identifient fortement aux Franco-Ontariens soulignent l'importance de l'Église catholique dans l'histoire de l'Ontario français notamment par la création d'écoles et l'organisation des paroisses qui ont assuré la survie du fait français ${ }^{27}$ :

La lutte pour un système scolaire franco-ontarien ne fait que commencer. Dans l'Ontario français, l'Église catholique devient le pilier du développement des " communautés franco-ontariennes " à travers la province. Ce sont 
les communautés religieuses qui vont créer/ouvrir les écoles et universités (1848 - Oblats fondent le Collège de Bytown, deviendra l'Université d'Ottawa; les Jésuites fondent le Collège du Sacré-Cour à Sudbury, etc.) De plus, les communautés francophones s'élèvent presque toutes à l'aube du clocher, c'est d'abord une paroisse avant d'être une ville. Se sont également les prêtrescolonisateurs vont recruter des francophones de l'Est ontarien pour aller coloniser le Nord, comme Hearst, Timmins, etc. (participant 006)

Cette reconnaissance d'une dette historique contractée envers l'Église catholique tranche avec les observations de Létourneau qui a remarqué que le récit des jeunes Québécois semble indifférent à cette dimension du passé canadien-français.

Enfin, la filiation entre le territoire, l'histoire, la culture et la langue française est présente dans le récit de plusieurs futurs enseignants. C'est une dimension essentielle du projet identitaire franco-ontarien qui retrace la présence française dès le début de la Nouvelle-France. Dans cet exemple, le participant considère que la province ontarienne a d'abord été française avant d'être occupée par les Britanniques avec la Conquête :

L'histoire de l'Ontario (écrite) débute dès l'arrivée des Français en Amérique du Nord, notamment avec les projets d'évangélisation des groupes autochtones en Huronie et en territoires Iroquoiens. Des Jésuites sont donc présents dans les territoires des Grands Lacs et vivent dans les diverses communautés autochtones - ici, j'évite les divers conflits et massacres. De plus, des voyageurs et coureurs des bois (par exemple Étienne Brûlé) travaillent dans ces régions éloignées. Certains s'installent et fondent des familles, tandis que d'autres font le pont entre les régions d'échange et de commerce, comme par exemple sur la rivière des Outaouais, tout près de l'emplacement du Parlement du Canada. [...] La colonisation du territoire actuel de l'Ontario était généralement française jusqu’à la Révolution Américaine. [...] Des colonies françaises, comme par exemple à Windsor et Détroit, deviennent des villes importantes économiquement pour la région. Avec la perte de la Nouvelle-France par la France en 1760, le territoire du sud de l'Ontario actuel passe en mains britanniques (le nord appartenant à la Compagnie de la Baie d'Hudson.) (participant 011)

En conclusion, est-ce que les futurs enseignants sondés peuvent être considérés comme des passeurs culturels et des ambassadeurs de l'identité franco-ontarienne? Ils adhèrent et adoptent pour la plupart un récit identitaire franco-ontarien qui se caractérise par la colonisation et l'exploration française, l'enracinement des populations francophones sur le territoire ontarien et surtout les luttes épiques pour le fait français (Règlement XVII et l'Hôpital Montfort). Toutefois, est-ce que ce récit identitaire se limite à une conviction personnelle ou, au contraire, se trouve-t-il au cœur de l'engagement pédagogique, professionnel et citoyen de ces futurs enseignants? Une question, sur laquelle nous nous pencherons plus loin, qui renvoie au processus de formation professionnelle, mais aussi aux attentes formelles et informelles du système scolaire envers ces futurs enseignants. 


\section{Les élèves au secondaire : " savoir sans connaître " ${ }^{28}$}

Comme nous l'avons souligné précédemment, les récits des élèves du secondaire sont nettement plus circonscrits que ceux des futurs enseignants - tant en terme de longueur que de teneur. Par ailleurs, devant leur incapacité à raconter un récit sur l'Ontario, certains élèves $(26 \%)$ déclarent forfait :

Malheureusement je ne me souviens pas beaucoup de l'histoire de l'Ontario comme tel et il me faudrait un cours de révision spécifiquement pour revoir l'histoire de la province. Je me souviens de nombreux évènements lié au Canada, mais je ne voudrais pas me tromper en attribuant un évènement du Québec (par exemple) à quelque chose qui serait arrivé en Ontario. Donc je préfere afin d'éviter les erreurs ne rien dire au sujet de mes connaissances historique sur l'Ontario. (questionnaire 23.04)

Honnêtement, je ne me rappelle de rien. Mais je pense que, il y avait beaucoup de troubles avec les français canadien et les anglais canadien de l'Ontario. Et je connais lime nationale par cœur.. (-). Oh et je sais que Mackenzie King étais le premier ministre a un point et c'est pour cela que nous avons un pont qui se nomme Mackenzie King... (questionnaire 38)

Ceci étant dit, plusieurs jeunes ont néanmoins produit des récits d'histoire riche de sens et d'intérêt. Comme l'illustre le tableau 3, leurs récits comportent un nombre important d'éléments factuels qui orientent leur narration. Ainsi, les mots qui reviennent le plus souvent dans les récits: Ontario (81\%), Canada (79\%), la province (46\%), Français (42\%), histoire (42\%), Anglais (42\%), Québec (33\%) et Francophone $(32 \%)$. Dans un moins grand pourcentage de cas, mais tout de même significatif, on retrouve une série de mots clés qui réferent à la guerre (30\%), à Ottawa (28\%), au Bas-Canada (21\%), aux Franco-Ontariens (21\%), à Champlain (18\%), au Haut-Canada (18\%), à l'école (18\%), au Règlement XVII, à Montfort (16\%) et Gisèle Lalonde (14\%) et à Étienne Brûlé (12\%).

Tableau 3

Mentions factuelles dans les récits selon le pourcentage de cas

\begin{tabular}{|c|c|}
\hline Mots employés au secondaire & $\begin{array}{c}\text { Pourcentage de cas } \\
\text { (n=58 èlèves du secondaire) }\end{array}$ \\
\hline ONTARIO & $81 \%$ \\
\hline CANADA & $79 \%$ \\
\hline PROVINCE & $46 \%$ \\
\hline FRANÇAIS & $42 \%$ \\
\hline HISTOIRE & $42 \%$ \\
\hline ANGLAIS & $42 \%$ \\
\hline OUÉBEC & $33 \%$ \\
\hline
\end{tabular}




\begin{tabular}{|c|c|}
\hline Mots employés au secondaire & $\begin{array}{c}\text { Pourcentage de cas } \\
\text { ( } n=58 \text { élèves du secondaire) }\end{array}$ \\
\hline FRANCOPHONE & $32 \%$ \\
\hline GUERRE & $30 \%$ \\
\hline OTTAWA & $28 \%$ \\
\hline PAYS & $28 \%$ \\
\hline VILLE & $23 \%$ \\
\hline FRANCO (ONTARIEN) & $21 \%$ \\
\hline BAS-CANADA & $21 \%$ \\
\hline CHAMPLAIN & $18 \%$ \\
\hline TERRITOIRE & $18 \%$ \\
\hline HAUT-CANADA & $18 \%$ \\
\hline ÉCOLE & $18 \%$ \\
\hline RÈGLEMENT XVII & $16 \%$ \\
\hline MONTFORT & $16 \%$ \\
\hline CULTURE & $16 \%$ \\
\hline CAPITALE & $16 \%$ \\
\hline AUTOCHTONES & $16 \%$ \\
\hline BAS CANADA & $16 \%$ \\
\hline ANGLOPHONE & $14 \%$ \\
\hline GISÈLE LALONDE & $14 \%$ \\
\hline DROIT & $14 \%$ \\
\hline LANGUE & $14 \%$ \\
\hline ÉTATS-UNIS & $14 \%$ \\
\hline ÉTIENNE BRÛLÉ & $12 \%$ \\
\hline POUVOIR & $11 \%$ \\
\hline
\end{tabular}

À la lumière de ce tableau, on remarque encore ici l'importanc-e accordée au territoire (Ontario, Canada) et aussi, dans une certaine mesure, aux trois moments forts qui jalonnent l'histoire franco-ontarienne : la colonisation française du pays (Samuel de Champlain ou Étienne Brûlé, les Autochtones), l'affrontement contre la majorité anglaise lors du Règlement XVII (les écoles francophones) et la lutte pour sauvegarder l'Hôpital Montfort (Hôpital Montfort, Gisèle Lalonde).

Samuel de Champlain est venu avec Étienne Brûlé pour coloniser le Nouveau Monde. (questionnaire 032)

Samuel de Champlain et Jacques Cartier ont découvert quelque chose dont je ne me souviens plus. Béatrice Desloges avait des écoles clandestines francophones et s'est battue avec des épingles contre des policiers pour abolir le règlement 17. Gisèle Lalonde qui s'est contre la fermeture de l'hôpital Montfort. (questionnaire 047) 
Fait intéressant, le récit des élèves s'inscrit souvent dans une perspective dualiste, soit de conflit entre les puissances coloniales ou entre des groupes identitaires (les Français, les Anglais, le conflit anglo-franco, la Conquête, les droits, la langue, etc.).

En 1917, je crois, le gouvernement a créé le règlement 17 qui interdit l'enseignement en français dans les écoles en Ontario. Grâce entre autres aux hérö̈ques des mères et de femmes associés à l'école Guigues, ici à Ottawa, qui ont mis la pression sur le gouvernement pour rétablir l'enseignement en français ici en Ontario. (questionnaire 023)

Ontario faisait anciennement partie de "Nouvelle-France ", mais les français et anglais se sont battus pour les terres de Ontario et Québec pour plusieurs années aides par des tribus autochtones. Finalement on s'est fait un gouvernement qui a réglé les disputes avec quelques exceptions (Louis Riel, franco-ontariens, anglo-québécois) et a divise les deux terres. Donc, il y a des gens d'orginie français et anglais qui habitent en Ontario. (questionnaire 026.04)

Souvent, malgré une démission apparente (rappelons que $26 \%$ s'avouent initialement vaincus par la question), les élèves dévoilent que les seuls aspects de l'histoire de l'Ontario dont ils se souviennent sont liés au caractère dualiste et conflictuel de l'évolution de la société :

Tout d'abord, je ne me souviens pas vraiment de ce que j'ai appris pour l'histoire de l'Ontario. Tout ce dont je me souviens, c'est que les anglais et les français ont fait une grande guerre, ayant pour but de coloniser le Canada. Les anglais on réussit à battre les français. Grace à cette victoire, les anglais colonisent l'Ontario. Malgré cela, il resta quand même des francophones dans la province. Tous ces francophones n'eurent pas le choix de s'adapter au système anglais. Ils n'eurent aussi pas le choix d'apprendre à parler l'anglais. Ceux qui refusaient se fessaient tuer, ou étaient chassés du pays. D'où l'on connait les Acadiens. (questionnaire 040)

Je ne me rappelle pas trop de l'histoire de l'Ontario particulièrement, mais je sais que c'était le centre commercial du Canada pendant les années 1800. Je me rappelle également que l'Ontario était une province majoritairement anglophone. Alors, les franco-Ontariens devaient se battre pour étudier en français. (questionnaire 046)

Tout comme pour notre échantillon de futurs enseignants, nous avons croisé les données du contenu factuel des récits avec le sentiment d'appartenance tel que rapporté par les élèves. À l'instar des universitaires, les 18 élèves du secondaire qui s'identifient fortement aux Franco-Ontariens sont ceux qui utilisent le plus de mots pour raconter l'histoire. Le tableau 4 illustre que certains mots clés sont fortement associés au sentiment d'appartenance des jeunes vis-à-vis la communauté franco-ontarienne, tels 
les termes " nous ", " notre ", " culture ", " histoire ", " école " et " Montfort ". Ces termes sont révélateurs d'une certaine conscience historique et contribuent à poser le cadre d'un récit marqueur de sens qui permet aux participants de puiser dans le passé pour se forger une identité.

Tableau 4

Nombre de mentions thématiques dans les récits selon le nombre de participants

\begin{tabular}{|c|c|c|c|}
\hline Mots clés & $\begin{array}{c}\text { Identité franco- } \\
\text { ontarienne forte } \\
(\mathbf{n}=\mathbf{1 8})^{\mathbf{2}}\end{array}$ & $\begin{array}{c}\text { Identité franco- } \\
\text { ontarienne } \\
\text { moyenne à faible } \\
\text { (n=35) }\end{array}$ & $\begin{array}{c}\text { Identification } \\
\text { prédominante }\end{array}$ \\
\hline NOTRE & 20 & 0 & Forte \\
\hline NOUS & 18 & 6 & Forte \\
\hline CULTURE & 13 & 3 & Forte \\
\hline GOUVERNEMENT & 9 & 2 & Forte \\
\hline ANGLETERRE & 8 & 2 & Forte \\
\hline HISTOIRE & 26 & 23 & Forte \\
\hline MONTFORT & 8 & 3 & Forte \\
\hline ÉCOLE & 10 & 5 & Forte \\
\hline OTTAWA & 7 & 5 & Forte \\
\hline BAS & 17 & 19 & $\begin{array}{c}\text { Moyenne } \\
\text { à faible }\end{array}$ \\
\hline PROVINCE & 2 & 11 & Forte \\
\hline CAPITALE & 5 & 83 & Moyenne \\
\hline ON faible
\end{tabular}

2. Le nombre total de réponses n'arrive pas à 58 car cinq des participants n’ont pas complété la section du questionnaire traitant de l'identité. 
Voici un extrait de récit politiquement engagé produit par un élève qui s’identifie fortement aux Franco-Ontariens; ce récit contient plusieurs des mots clés présentés dans les tableaux 3 et 4 :

Le français au canada étais la langue principale au débuts mes cela a changer quand le pays est aller du contrôle de la France a celle de la Grande Bretagne cependant des endroit francophone on rester telle le Québec, Montréal et autre personne a travers le Canada. A un point il on voulue abolir les écoles français et des femme comme Béatrice-Désloge et Jeanne Sauver on apprit au enfant le français en secret. La communauté francophone c'est aussi rassembler quand on a voulue fermer l'hôpital Monfort une des ceule ou la seule hôpital francophone de la région et le peuple c'est rebeller. (questionnaire 067)

À l'inverse, ceux s'identifiant moyennement ou peu à l'Ontario français $(n=35)$ ont davantage utilisé des termes vagues et descriptifs comme " capitale », "Francophone " et "Québec ». Ces thèmes ont souvent peu de charges émotives ou identitaires et ne possèdent pas de caractère engagé, comme l'illustrent les passages suivants:

L'Ontario est une des 13 provinces du Canada situé entre le Québec et le Manitoba, la capitale de l'Ontario est Toronto. L'Ontario contient la capitale nationale du Canada ; Ottawa. Sir John A. Macdonald était le premier premier ministre du Canada. Sir Wilfrid Laurier était aussi un premier ministre canadien plus tard. Aujourd'hui Stephen Harper est le premier ministre du Canada. Le drapeau de l'Ontario est rouge avec le drapeau britannique dans le coin en haut à gauche et 3 feuilles d'érables a la droite. (questionnaire 070)

Autre fait intéressant, les élèves s'identifiant moyennement ou peu à l'Ontario français ont également rédigé leur récit en faisant plus souvent appel à la première personne du singulier (le « je ") plutôt qu'au "nous " collectif; ce "nous » qui renvoie à une communauté au sein de la francophonie de l'Ontario. Par ailleurs, le « je " sert souvent à exprimer leur incapacité à formuler un récit substantiel sur l'Ontario.

[...] en fréquentant des écoles francophones durant toute ma vie, j’ai appris plus profondément au sujet de ma culture francophone. Comment mes ancêtres en Ontario se sont battues pour que je puisse poursuivre une éducation en français. Je ne connait pas plusieurs éléments du passé que je jugerais être important dans l'histoire de l'Ontario [...]. (questionnaire 036)

Enfin, les élèves avec un faible sentiment d'appartenance aux Franco-Ontariens inscrivent leur récit beaucoup plus souvent dans une trame présentiste, c'est-à-dire qu'ils réferent davantage aux conséquences contemporaines de l'histoire qu'à ses causes plus anciennes : souvent en association aux avantages du bilinguisme et du multiculturalisme qu'offre la province et la capitale fédérale en particulier : 
Nous ici en Ontario, nous sommes très choyer d'avoir un pays multiculturel, car sa nous ouvre des portes a plusieurs travail. En Ontario nous avons plusieurs droits. (questionnaire 042.2)

Pour en revenir à la fierté Ontarienne, je pense que ce qui l'a causé c'est le système éducatif et les opportunités que nous avons ici. L’Ontario offre beaucoup et je suis consciente de plusieurs organisations pour aider sa population. (questionnaire 063)

Force est de constater que ce ne sont pas tous les élèves sondés qui ont un degré d'engagement envers l'aventure historique de l'Ontario français. Ainsi, bien que certains présentent volontiers des connaissances historiques en lien avec des moments forts de cette communauté, ils le font toutefois de manière descriptive sans les rattacher à des convictions personnelles, comme le résume assez bien l'élève suivant, qui s'identifie peu aux Franco-Ontariens : "Je connais aussi en résumé l'histoire de l'école Guiges et du règlement 17. Tout ce que je sais sur l'histoire des Franco-Ontariens je l'ai retenu des spectacles qu'on voit pour la fête des Franco Ontariens en Septembre » (questionnaire 065).

\section{Discussion}

Que peut-on établir comme bilan provisoire à partir des résultats de cette étude exploratoire visant à sonder la conscience historique des jeunes francophones de l'Ontario? Notre étude est porteuse et pertinente dans la mesure où elle présente un premier coup de sonde offrant un portrait unique de la conscience historique des jeunes d'Ottawa fréquentant le système scolaire franco-ontarien. Elle nous offre des balises importantes pour la suite de notre étude à l'échelle provinciale. Il faut néanmoins rappeler la prudence avec laquelle nous devons interpréter les résultats des élèves et des futurs enseignants de la région d'Ottawa. L'Ontario français est composé d'une population francophone de plus en plus diversifiée sur les plans social et culturel. Ainsi, les francophones de la capitale fédérale ne sont pas nécessairement représentatifs des communautés francophones des régions de l'est, du nord-est, du centre ou du sud-ouest de l'Ontario.

Le premier constat qui émane de notre recherche exploratoire est qu'il est faux de prétendre que les jeunes Ontariens sont totalement amnésiques ou ignorants du passé de leur communauté. Néanmoins, on peut être critique de l'étendue ou de l'exactitude de leurs connaissances historiques, souvent limitées et simplistes. Or, chez la plupart des jeunes sondés, leur vision du passé demeure organisée, cohérente et porteuse de sens. Pour reprendre l'expression de Létourneau, beaucoup d'élèves de notre enquête " savent sans connaître ". ${ }^{29}$ C'est-à-dire qu'ils possèdent certaines notions historiques et peuvent efficacement les transposer dans un récit liant le passé au présent, mais qui comporte toutefois plusieurs inexactitudes et, à l'occasion, une certaine confusion. Si les participants de notre étude ne sont clairement pas des experts en la matière (quelques-uns avouant carrément ne plus se souvenir de rien), ils 
ont néanmoins complété l'ensemble du cursus scolaire obligatoire et possèdent des connaissances historiques de l'Ontario et plus particulièrement de l'Ontario français.

Fait intéressant, malgré la diversité des niveaux d'enseignement chez les élèves de l'enquête, les éléments clés véhiculés par leurs récits ont beaucoup en commun. Ils mettent en relief la filiation entre le territoire, l'histoire, la culture et la langue française et ce, même si la question de recherche portait sur l'Ontario et non pas directement sur l'Ontario français. De plus, il est frappant de constater comment les étudiants à l'université et les élèves au secondaire placent l'école au cœur de l'expérience historique et de l'identité franco-ontarienne. L'école et les luttes scolaires apparaissent comme un catalyseur, particulièrement chez les jeunes qui s'identifient fortement aux Franco-Ontariens, qui révèle la volonté de "vivre " de cette communauté, de se perpétuer dans l'avenir et de transmettre aux générations suivantes un héritage marqueur de sens.

Les jeunes tirent vraisemblablement leur inspiration de ce qu'ils ont appris lors des cours d'histoire à l'élémentaire et au secondaire au sein du système scolaire franco-ontarien. Ainsi, plusieurs éléments rapportés par les jeunes (p. ex. : Samuel de Champlain, Étienne Brûlé, le Haut-Canada, le Bas-Canada, le Règlement XVII) se retrouvent textuellement dans les contenus d'apprentissage des programmes-cadres. En ce sens, à la lumière de nos résultats, l'école franco-ontarienne joue bel et bien un rôle dans l'éducation historique de ces jeunes. Elle permet à des élèves provenant de cultures, de langues et d'horizons variés de s'approprier une certaine compréhension de l'aventure historique franco-ontarienne.

Mais on ne saurait sous-estimer les connaissances acquises grâce au curriculum réel de l'élève, issu d'expériences diverses et fluctuantes lors de visites aux musées ou aux parcs, de discussions familiales, de visionnement de films, de rencontre entre amis ou de participation à des activités sociales ou communautaires. On peut penser ici aux nombreuses références dans les récits des jeunes à l'hôpital Montfort, seul hôpital francophone ontarien, qui fut et demeure pour eux un symbole de la résistance afin de protéger leurs droits collectifs face aux velléités " assimilatrices » du gouvernement conservateur de Mike Harris. De même en est-il de l'ancienne mairesse de Vanier, Gisèle Lalonde — une école de la région d'Ottawa porte son nom —, qui représente pour plusieurs jeunes le porte-étendard de cette lutte de tous les instants pour les droits des Franco-Ontariens. À ce titre, nos résultats de recherche révèlent que les élèves utilisent les crises du Règlement XVII et de l'Hôpital Montfort comme les pôles structurants d'un récit qui définit leur rapport au passé et permet d'exprimer leurs aspirations culturelles et identitaires au temps présent. Cette analyse des événements fondateurs peut être transposée sur les figures héroïques de Béatrice Desloges et de Gisèle Lalonde qui incarnent la volonté collective des Franco-Ontariens face à l'adversité. ${ }^{30}$

Bien que nos résultats préliminaires doivent être approfondis, nous constatons que la vision que les élèves possèdent de l'histoire de l'Ontario est structurée par une conscience historique plus ou moins développée au gré d'apprentissages informels. Ces apprentissages ne sont pas toujours le résultat pur et simple de leur propre volonté d'apprendre dans le contexte formel et structuré de la salle de classe. Une 
partie de la conscience historique des jeunes est donc cachée dans la mesure où leurs expériences d'apprentissage ne sont pas toujours directement observables par les chercheurs ou même les enseignants eux-mêmes. ${ }^{31}$

Le deuxième constat de notre enquête auprès de jeunes d'Ottawa porte sur l'impact du sentiment d'appartenance envers la communauté franco-ontarienne et la narration de l'histoire de l'Ontario français. Les résultats de l'étude montrent clairement que les élèves qui s'identifient fortement aux Franco-Ontariens ont produit des récits historiques plus riches et engagés que les autres. Ces élèves ont fait appel à des référents factuels et des mythes fondateurs de l'Ontario français (p. ex. : les explorateurs, l'Église catholique, les écoles francophones, le Règlement XVII, la culture, les luttes, Montfort, la communauté, le "nous »). Ces jeunes francophones connaissent et savent mobiliser efficacement certains "noyaux énonciatifs basiques " propres au régime historial et mémoriel de la communauté d'appartenance franco-ontarienne. ${ }^{32}$ Les multiples références à Étienne Brûlé comme " premier Franco-Ontarien » illustre assez bien cet usage contemporain du passé à des fins d'orientation collective permettant la continuité du groupe dans l'évolution de l'histoire humaine et des rapports entre francophones et anglophones. ${ }^{33}$ Comme le récit suivant l'indique :

L'histoire franco-ontarienne est marquée par plusieurs événements et plusieurs personnages historiques importants. Entre autre, Étienne Brûlé, qui, selon plusieurs est le véritable premier Franco-Ontarien. Il ne va sans dire que le Canada n'est pas un pays sans rupture. En effet, dès sa colonisation, il eut une séparation claire entre les Canadiens-français et les Canadiens-anglais. C'est une guerre constante entre la France et l'Angleterre pour coloniser et s'accaparer du territoire. (questionnaire 001)

Ces observations concernant l'usage du passé dans la construction identitaire des jeunes nous semblent confirmées par la comparaison entre les récits des élèves du secondaire et de ceux des futurs enseignants. Dans un cas comme dans l'autre, on retrouve chez les participants s'identifiant fortement à la communauté franco-ontarienne la même orientation narrative qui comporte une forme dualiste, c'est-à-dire qu'elle est caractérisée par les affrontements et les conflits, soit entre la France et l'Angleterre sous la période coloniale ou entre les Francophones et les Anglophones pour la période plus contemporaine. Plus précisément, la lutte pour une éducation en français est au cœur des récits dualistes, qui se décline la plupart du temps par les mots-clés suivants : guerre aux épingles, Béatrice Desloges, enseignement en français, Hôpital Montfort, Penetanguishene et Charte des droits et libertés.

Ce constat, présenté comme hypothèse dans tous les autres travaux sur la conscience historique des jeunes, permet donc d'affirmer sous toute réserve qu'il existe vraisemblablement au sein de cette société un cadre identitaire distinctif qui permet de façonner dans la conscience historique une façon toute particulière de mettre en récit le passé comme matrice de sens et d'orientation personnelle et collective. À l'évidence, le curriculum formel, mais aussi réel de l'élève, joue un rôle non négligeable sur son identité et sa conscience historique. Pour plusieurs, cette conscience est encore à une 
étape que l'on pourrait qualifiée de rudimentaire. Mais l'analyse des résultats indique que plus le jeune s'identifie fortement à son groupe d'appartenance, plus sa vision narrative du passé sera structurée et orientée selon la mémoire de l'aventure collective des Franco-Ontariens. Cette mémoire, qui présente l'Ontario français comme une communauté minoritaire qui définit son projet politique dans la logique du principe des "peuples fondateurs ", s'articule autour de trois moments forts. 1) L'exploration française avec les figures historiques de Champlain et surtout d'Étienne Brûlé, surnommé le " premier Franco-ontarien » qui fonde une société embryonnaire de langue française et de culture française. 2) La lutte pour une éducation en français lors de la crise du Règlement XVII et la victoire symbolique des Franco-Ontariens qui parviennent à faire respecter leurs droits par le gouvernement de l'Ontario et la majorité anglophone. 3) Enfin, le combat pour préserver l'Hôpital Montfort, alors que les Franco-Ontariens réussissent une fois de plus à écarter la menace qui pesait sur leurs droits fondamentaux, et qui appelle à la vigilance face aux dangers d'assimilation qui pèsent toujours sur la communauté franco-ontarienne.

Comme troisième constat, il n'est pas surprenant d'observer que les futurs enseignants sondés ont produit un récit beaucoup plus long, riche et porteur que les élèves du secondaire. Ces futurs enseignants font appel à un contenu historique étendu et ils présentent de nombreux d'événements, personnages et concepts propres à l'Ontario français. ${ }^{34}$ Par rapport aux élèves du secondaire, leurs récits contiennent quatre fois plus de mots et sont aussi beaucoup plus détaillés, détachés et critiques de l'histoire ontarienne et franco-ontarienne. On peut donc poser l'hypothèse que la formation académique des futurs enseignants, composée de plusieurs cours universitaires en histoire, mais aussi en didactique de l'histoire, joue un rôle vital dans la formation de leur conscience historique. Cette formation académique a su fournir à ces futurs enseignants de l'épaisseur et de la complexité à leur vision de l'histoire de l'Ontario.

\section{Conclusion : \\ Implications pour l'éducation des jeunes francophones de l'Ontario}

Les enquêtes comme la nôtre auprès des jeunes remplissent plusieurs fonctions. Elles dressent un portrait de l'état des connaissances et surtout des visions de l'histoire au sein d'une population donnée. Elles servent également à mesurer l'influence possible de l'école sur l'apprentissage historique et à formuler des recommandations auprès des instances éducatives pour améliorer le curriculum scolaire et les pratiques d'enseignement. Enfin, ces enquêtes permettent de nuancer les affirmations maintes fois répétées à propos de la jeunesse et son inculture généralisée à l'égard de l'histoire nationale. ${ }^{35}$ Chacune de ces fonctions est abordée dans nos conclusions. Enfin, en tant que spécialistes de l'éducation et de l'histoire de l'éducation, nous croyons au potentiel de notre enquête comme outil pédagogique servant de moyen d'évaluation diagnostique en appui à l'apprentissage chez les élèves.

Les enquêtes comme la nôtre sont rarement utilisées en classe. Autrement dit, elles ne sont pas transposées sous la forme d'activité pédagogique. Pourtant, ces enquêtes évaluent les connaissances préalables de l'histoire : travailler à partir de ces 
connaissances est un objectif de nombreux programmes d'histoire, dont celui de l'Ontario. Les connaissances préalables sont le bagage d'idées et de représentations avec lequel l'élève entre en classe et appréhende la matière enseignée. Ces connaissances proviennent aussi bien de la salle de classe que des apprentissages plus informels au gré d'expériences multiples. Des chercheurs comme VanSledright insistent pour que les enseignants prennent acte des connaissances préalables détenues par leurs élèves, de manière à confronter celles-ci efficacement. ${ }^{36}$ Plus particulièrement, le réputé didacticien Sam Wineburg suggère de discuter en classe avec les élèves des différentes sources de leur connaissance préalable de l'histoire, de manière à ce que ces derniers prennent conscience de la nécessité de critiquer et de bonifier ces sources. ${ }^{37}$ Même si plusieurs enquêtes traitent des connaissances historiques des élèves, peu de transpositions pédagogiques sont disponibles à leur sujet. Comme l'a révélé une étude pilote auprès de notre échantillon de futurs enseignants, il est possible d'aiguiser le sens critique des jeunes à partir d'une discussion sur leurs connaissances préalables et ainsi de favoriser une conscience historique plus réflexive.

Lors des entretiens de groupe tenus après la collecte de données, nous avons incité les futurs enseignants à exposer les sources d'inspiration de leur récit d'histoire de l'Ontario. Plusieurs nous ont confié avoir consciemment pris une distanciation idéologique par rapport à la mémoire collective ontarienne en écrivant leur récit d'histoire. Cette distance critique provenait pour la plupart de la formation reçue à l'université. Cette discussion a fait surgir des questionnements quant au rôle des professeurs d'histoire dans un contexte où les francophones sont un groupe minoritaire en Ontario. Dès lors, certains ont explicitement parlé du fait que leur rôle n'est pas d'endoctriner les jeunes dans une vision mémorielle du passé collectif franco-ontarien, mais plutôt de leur fournir aux élèves les outils méthodologiques, ainsi qu'une pensée historique nécessaire pour évaluer les divers récits du passé auxquels ils sont confrontés. Cette activité visait l'acquisition des compétences du praticien réflexif, celui-ci étant capable d'un jugement professionnel lucide et intelligent, tout autant qu'il est un être " désireux et capable de se prendre pour objet de sa réflexion, de " 'marcher et se regarder marcher" ". ${ }^{38}$ Ces futurs enseignants ont donc fait preuve d'objectivation et d'abstraction réfléchissante dans la mesure où ils ont su, avant et après notre enquête, réfléchir sur leur propre version de l'histoire de l'Ontario.

Cette étude exploratoire a permis de valider diverses questions de recherche initiales, ainsi qu'offrir des balises importantes concernant l'étude de la conscience historique de la jeunesse franco-ontarienne. Dans une prochaine étape, nous comptons élargir notre échantillonnage à l'extérieur d'Ottawa de manière à obtenir un portrait plus riche des diverses histoires qui façonnent la vision mais aussi l'identité des futurs citoyens de l'Ontario français. En effet, bien que la ville d'Ottawa constitue un théâtre d'expérimentation riche en potentiel d'analyse et d'interprétation, il reste qu'elle ne reflète pas nécessairement la variété et la complexité des autres milieux de vie francophone en Ontario qui favorise sans doute un autre rapport au passé. Ces études permettront non seulement de constituer une " cartographie » de la conscience historique des futurs citoyens francophones de l'Ontario, mais pourront servir de matière première à la création d'outils pédagogiques. 
Dans le contexte scolaire actuel, les élèves n'ont à peu près pas à réfléchir sur leurs propres conceptions du passé collectif et plus particulièrement, celle portant sur l'Ontario français. Ainsi, ils n’ont pas à développer les compétences nécessaires pour critiquer leur récit d'histoire et façonner ainsi une conscience historique plus poussée et réflexive. D'ailleurs, les jeunes auront à faire face à une pluralité de récits historiques dans leur vie personnelle et professionnelle dans une société canadienne caractérisée par une fluidité des identités. D'ailleurs, l'enquête nationale Les Canadiens et leurs passés, menée par Jocelyn Létourneau et son équipe auprès de la population canadienne, révèle que de plus en plus de récits d'histoire font compétition dans l'espace public canadien. Ils sont produits par les autorités politiques, mais aussi par plusieurs organismes et groupes de pression qui revendiquent une certaine crédibilité historique. Dès lors, les citoyens doivent être bien outillés pour juger de la qualité et de la pertinence de toutes ces affirmations à propos du passé et de la mémoire, ${ }^{39}$ en commençant par le récit d'histoire auquel il adhère. La mobilisation en classe d'histoire d'enquête comme la nôtre est une approche prometteuse pour engager les élèves francophones sur une voie scolaire rarement empruntée, mais utile tout au long de leur vie : l'évaluation du récit d'histoire, celui qui donne sens à leur vie et oriente leurs actions dans le temps. La prochaine étape de notre projet est donc d'enrichir notre corpus afin de valider nos résultats préliminaires et ainsi offrir aux enseignants et à la communauté un portrait plus vaste et complet de la conscience historique des jeunes francophones de l'Ontario.

\section{Notes}

1 Nous tenons à remercier la Faculté d'Éducation de l'Université d'Ottawa et le Centre de recherche en civilisation canadienne-française (CRCCF) pour leur soutien financier qui ont rendu possible cette recherche.

2 Ministère de l'Éducation de l'Ontario, Études canadiennes et mondiales, $11^{e}$ et $12^{e}$ année (Toronto : Imprimeur de la Reine pour l'Ontario, 2005), 269.

3 Au sujet de la contribution des historiens au récit fondateur franco-ontarien, voir les ouvrages suivants : Robert Choquette, L'Ontario français, historique (Montréal : Éditions Études Vivantes, 1980); Gaétan Gervais, "L'Ontario français, 1821-1910 ", dans Cornelius J. Jaenen (éd.), Les Franco-Ontariens (Ottawa : Les Presses de l'Université d'Ottawa, 1993), 49-126; Gaétan Gervais, "Aux origines de l'identité francoontarienne ", Cahiers Charlevoix, Études franco-ontariennes, 1 (Sudbury : Société Charlevoix et Prise de parole 1995), 125-168 ; Gaétan Gervais, «L'histoire de l'Ontario français, (1610-1997)", dans Joseph-Yvon Thériault (dir.), Francophonies minoritaires au Canada : l'état des lieux, (Moncton : Éditions d'Acadie, 1995), 145-161 ; Michel Bock, Gaétan Gervais et Suzanne Arseneault (dir.), L'Ontario français : des Pays-d'enHaut à nos jours (Ottawa : Centre franco-ontarien des ressources pédagogiques, 2004). Voir aussi du côté de la sociologie, Danielle Juteau et Lise Séguin-Kimpton, « La collectivité franco-ontarienne : structuration d'un espace symbolique et politique ", dans Cornelius J. Jaenen (dir.), Les Franco-Ontariens (Ottawa : Presses de l'Université d'Ottawa, 1993), 273-313. Roger Bernard, De Québécois à Ontarois, La communauté franco-ontarienne (Hearst : Le Nordir, 1988). Denis Gratton, Production de la différence: le cas ontarois, thèse de doctorat (science politique), Université Laval, Sainte-Foy, 1990. Jean Lapointe, « L'historiographie et la construction de l'identité ontaroise », dans 
Simon Langlois, (dir.), Identité et cultures nationales. L'Amérique française en mutation (Sainte-Foy : Les Presses de l’Université Laval, 1995), 153-166.

4 Voir l'autobiographie de Gisèle Lalonde: Gisèle Lalonde, Jusqu'au bout (Ottawa : Le Nordir, 2003).

5 Pour une trame narrative franco-ontarienne, voir Serge Dupuis, 400 ans de vie politique en Ontario français, une initiative du RÉFO dans le cadre du $400^{\mathrm{e}}$ anniversaire de la présence française, avec l'appui de Patrimoine canadien, 2014.

6 Anne Gilbert, Espaces franco-ontariens, Essai (Ottawa : Prise de parole, 1988).

7 Profil de la communauté francophone de l'Ontario, 2009, Office des Affaires francophones de l'Ontario, 14. http://www.ontario.ca/fr/francophones/profil-de-la-communautefrancophone-de-lontario-2009 (page consultée le 29 mai 2014).

8 Ibid., 10. En 2011 les immigrants comptent pour huit pour cent de la population franco-ontarienne, vingt-neuf pour cent de la communauté francophone de Toronto et huit pour cent de celle d'Ottawa. Toutefois, ces données doivent être analysées prudemment puisqu'elles different du dernier recensement de 2006 en raison de l'adoption d'un nouveau questionnaire. Source : Statistique Canada, Division de la géographie, Recensement de la population de 2011.

9 Voir Gervais, «L'Ontario français, 1821-1910 »..., 56-64 et Philippe Orfali, « Ottawa, capitale de l'Ontario français ", Le Droit, 17 février 2013.

10 Rapport de la Commission royale sur l'éducation, Pour l'amour d'apprendre (Toronto : Imprimerie de la Reine pour l'Ontario, 1995). Voir le site du Ministère de l'Éducation de l'Ontario http://www.edu.gov.on.ca/fre/general/abcs/rcom/mainf.html (page consultée le 29 mai 2014).

11 Ministère de l'Éducation de l'Ontario, "L'éducation en langue française en Ontario, de quoi s'agit-il ? » L'éducation en langue française en Ontario. Disponible : http://www. edu.gov.on.ca/fre/amenagement/index.html

12 Ministère de l'Éducation de l'Ontario, Études sociales, de la $I^{r e} a ̀ ~ l a ~ G^{e}$ année (Toronto : Imprimeur de la Reine pour l'Ontario, 2013), 52.

13 Ministère de l'Éducation de l'Ontario, Études canadiennes et mondiales, $9^{\prime}$ et $10^{\circ}$ année (Toronto : Imprimeur de la Reine pour l'Ontario, 2013), 119.

14 Ibid., 127.

15 Ministère de l'Éducation de l'Ontario, Études canadiennes et mondiales, $11^{e}$ et $12^{\circ}$ année (Toronto : Imprimeur de la Reine pour l'Ontario, 2005), 269.

16 Il y a toutefois une étude sur le lien entre la mémoire et l'identité sociale des FrancoOntariens : Christian R. Bellehumeur, Francine Tougas et Joëlle Laplante, « Le devoir de mémoire : le lien entre la mémoire collective et l'identité sociale chez les FrancoOntariens ", Revue canadienne des sciences du comportement/Canadian Journal of Behavourial Science, vol. 41, 3 (2009) : 169-179.

17 Jocelyn Létourneau, Je me souviens? Le passé du Québec dans la conscience de sa jeunesse (Montréal : Fides 2014).

18 Jörn Rüsen, History: Narration, Interpretation, Orientation (New York : Berghahn Books, 2005).

19 Philippe Perrenoud, "Curriculum : le formel, le réel, le caché ", dans J. Houssaye, J. (éd.) La pédagogie : une encyclopédie pour aujourd'hui (Paris : ESF, 1993), 61-76.

20 Jocelyn Létourneau et Sabrina Moisan, "Mémoire et récit de l'aventure historique du Québec chez les jeunes Québécois d'héritage canadien-français : coup de sonde, amorce des résultats, questionnements ", The Canadian Historical Review, vol. 84, 2 (juin 2004) : 325-356; Jocelyn Létourneau et Christophe Caritey, «L'histoire du Québec racontée par les élèves de $4^{\mathrm{e}}$ et $5^{\mathrm{e}}$ secondaire : l'impact apparent du cours d'histoire nationale dans la structuration d'une mémoire historique collective chez les jeunes Québécois ", Revue d'histoire de l'Amérique française, vol. 62, 1 (2008) : 69-93; Stéphane Lévesque, Jocelyn Létourneau et Raphaël Gani, «'A giant with clay feet': 
Québec students and their historical consciousness of the nation ", International Journal of Historical Learning Teaching and Research, (May 2013) : 159-175; Marc Robichaud, "L'histoire de l'Acadie telle que racontée par les jeunes francophones du NouveauBrunswick: construction et de construction d'un récit historique ", Acadiensis, XL, 2 (été/automne 2011), 33-69. Voir aussi l'ouvrage récent de Jocelyn Létourneau, Je me souviens? Le passé du Québec dans la conscience de sa jeunesse, (Montréal : Fides 2014). (op. cit.)

21 D'autres études se sont penchées sur les récits historiques produits par les élèves pour comprendre leurs schémas narratifs : James Wertsch, "Specific Narratives and Schematic Narratives Templates ", dans Peter Seixas (dir.), Theorizing Historical Consciousness (Toronto : University of Toronto Press, 2004), 49-62 et James Wertsch et Kevin O'Connor, "Multivoicedness in Historical Representation : American College Student's Accounts of the Origins of the US ", Journal of Narrative of Life History, vol. 4, 4 (1994): 295-309.

22 Nous avons privilégié un récit sur l'Ontario plutôt que l'Ontario français et même l'histoire du Canada pour plusieurs raisons. En effet, la notion d'Ontario français est beaucoup plus récente que l'Acadie qui comporte une charge identitaire très forte inscrite dans l'histoire et ne constitue pas une entité politique proprement dite comme le Québec qui peut se réclamer d'un nationalisme et s'appuyer sur un nombre important d'institutions publiques et étatiques. De plus, l'Ontario français est un terme qui peut prêter à la controverse et ne suscite pas toujours l'unanimité même parmi les francophones. Il peut même revêtir un biais idéologique contrairement à une question centrée sur l'histoire de l'Ontario qui apparait plus neutre et qui peut inclure une histoire de l'Ontario français, alors que l'inverse paraît moins probable.

23 L'échelle de mesure est décrite en détail dans l'article d'Arthur Aron, Elaine N. Aron, et Danny Smollan, "Inclusion of Other in the Self Scale and the structure of interpersonal closeness ", Journal of Personality and Social Psychology, 63, 4 (Oct. 1992): 596-612.

24 L'échantillon comportait dix-huit étudiants en didactique de l'histoire à l'Université d'Ottawa. Cinq d'entre eux ont participé à la question « Ontario français » et treize à la question «Ontario ». L'échantillon comprenait neuf Québécois, sept Ontariens, un Français et un Sénégalais, ainsi que dix hommes et huit femmes. Enfin, la moyenne d'âge était d'environ 26 ans.

25 L'évaluation de l'appartenance envers la communauté franco-ontarienne est caractérisée par une série de sept images qui dépeignent chacune un couple de cercles qui s'enchevêtre à différent niveau. Dans un cercle est écrit la mention moi, et dans l'autre Franco-ontarien. La première image représente deux cercles symétriquement opposés - une dissociation entre le " moi » et les Franco-Ontariennes — et la dernière image $\left(7^{\mathrm{e}}\right.$ de 7$)$ représente deux cercles superposés : une fusion entre le « moi » et les Franco-Ontarien ".

26 Dans un prochain article, nous analyserons la structure narrative du récit de manière à mieux évaluer l'orientation particulière qu'ont donnée les jeunes à leur histoire de l'Ontario et ainsi entrer au cœur de leur vision narrative du passé en lien avec le présent et l'avenir possible.

27 L'historien Robert Choquette a bien mis en lumière le rôle fondateur de l'Église catholique dans la constitution d'une communauté de langue, de religion et de mémoire. Robert Choquette, L'Église catholique dans l'Ontario français du dix-neuvième siècle (Ottawa : Éditions de l'Université d'Ottawa, 1984).

28 Les notions de " connaissance » et de "savoir " sont souvent confondues dans le discours populaire. Or, il existe des différences importantes. Les connaissances font références à un ensemble établi d'éléments propres à une matière (faits, termes, définitions, etc.), alors que le savoir est plutôt le résultat d'une production selon certaines conventions sociales et qui est sujet aux révisions et réévaluations. Le savoir est le fruit d'une certaine 
interaction avec la matière et entre des sujets, le fruit d'une interaction langagière dans un contexte particulier (par ex. : un contexte professionnel ou disciplinaire). Le savoir comporte donc des dimensions discursive, communicative et argumentative. Voir à ce sujet Clermont Gauthier (dir.), Pour une théorie de la pédagogie : recherches contemporaines sur le savoir enseignant, (Québec : Presses de l'Université Laval, 1997), chap. 6.

29 Létourneau, Je me souviens?..., op. cit., 18.

30 Les sœurs Diane et Béatrice Desloges peuvent être considérées comme des icônes dans l'imagerie populaire puisqu'elles ont refusé d'appliquer le Règlement XVII visant à limiter l'enseignement du français, déclenchèrent une grève avec la participation de leurs élèves et instaurèrent plus tard des classes clandestines. Lorsque les autorités tentèrent de les en chasser, ils furent repoussés par les institutrices Desloges, armées de leurs épingles à chapeau, et les parents qui soutenaient leur action. Voir le site du Réseau du patrimoine franco-ontarien: http://www.rpfo.ca/en/Biographies_40/Les-Soeurs-DianeDesloges-18921945-Et-Beatrice-Desloges-18951957_126.

31 Philippe Perrenoud, "Mettre la pratique réflexive au centre du projet de formation ", Cahiers pédagogiques, 390 (2001) : 42-45.

32 Létourneau et Caritey, "L'histoire du Québec racontée par les élèves de $4^{\mathrm{e}}$ et $5^{\mathrm{e}}$ secondaire", loc. cit., 91.

33 Au sujet de la construction identitaire autour de la figure historique d'Étienne Brûlé, voir Jean-François Beaudet, Étienne Brûlé (Montréal : Lidec, 1993) et Stéphanie SaintPierre, "Étienne Brûlé : la création d'un personnage ", Revue du Nouvel-Ontario, 29 (2004) : 5-44.

34 Il faut le rappeler, près de la moitié des futurs enseignants sondés provenaient de la province de Québec.

35 Voir, par exemple, les sondages menés auprès de la population lors des jours commémoratifs (Jour du Souvenir, fête du Canada, etc.) par des organismes tels que l'Institut du Dominion/Historica : https://www.historicacanada.ca/sites/default/files/ PDF/polls/remembrance2008_fr.pdf (page consultée le 1er juin 2014).

36 Bruce A. VanSledright, The Challenge of Rethinking History Education. On Pratices, Theories and Policy (New York : Routledge, 2011), particulièrement le chapitre 2.

37 Sam Wineburg et son équipe ont interrogé des jeunes à propos de la guerre du Vietnam, pour constater à leur grande surprise que Forrest Gump constituait pour plusieurs d'entre eux une source historique de référence. Ainsi, cette équipe de recherche a réfléchi à la manière d'utiliser en salle de classe ce résultat provenant d'une enquête similaire à la nôtre. Ils suggèrent de questionner les jeunes à propos de leur récit d'histoire ainsi que des sources à sa base. Comme le soulignent Wineburg et son équipe, «By asking students why they think they have retained such a vivid image of Forrest hailing Jenny from the podium but have no idea how he found himself on that podium in the first place, educators can turn a cultural product like Forrest Gump on its head ». Sam Wineburg, et al., "Forrest Gump and The Future of Teaching the Past», Phi Delta Kappan, 89, 3, (Nov 2007) : 175.

38 Perrenoud, "Curriculum : le formel, le réel, le caché » loc. cit., 42-45.

39 Margaret Conrad et al., Canadians and Their Pasts (Toronto : University of Toronto Press, 2013), 153. 